\title{
Autologous Antibodies that Bind Neuroblastoma Cells
}

Yujing Sun, ${ }^{a}$ Giselle S. Sholler, ${ }^{\mathrm{b}}$ Girja S. Shukla, ${ }^{\mathrm{a}}$ Stephanie C. Pero, ${ }^{\mathrm{a}}$ Chelsea L.Carman, ${ }^{\mathrm{a}}$ Ping Zhao, ${ }^{\mathrm{b}}$ David N. Krag ${ }^{\mathrm{a}, 1}$

a Department of Surgery, Vermont Cancer Center, University of Vermont College of Medicine, Burlington, VT 05405, USA

b Helen DeVos Children's Hospital and Michigan State University, Grand Rapids, MI 49503, USA

1 Corresponding Author: Department of Surgery, Vermont Cancer Center, University of Vermont College of Medicine, Burlington, VT 05405, USA. Tel.: 802656 5830; fax: 802656 5833. E-mail address: david.krag@uvm.edu

\begin{abstract}
:
Antibody therapy of neuroblastoma is promising and our goal is to derive antibodies from patients with neuroblastoma for developing new therapeutic antibodies. The feasibility of using residual bone marrow obtained for clinical indications as a source of tumor cells and a source of antibodies was assessed. From marrow samples, neuroblastoma cells were recovered, grown in cell culture and also implanted into mice to create xenografts. Mononuclear cells from the marrow were used as a source to generate phage display antibody libraries and also hybridomas. Growth of neuroblastoma patient cells was possible both in vitro and as xenografts. Antibodies from the phage libraries and from the monoclonal hybridomas bound autologous neuroblastoma cells with some selectivity. It appears feasible to recover neuroblastoma cells from residual marrow specimens and to generate human antibodies that bind autologous neuroblastoma cells. Expansion of this approach is underway to collect more specimens, optimize methods to generate antibodies, and to evaluate the bioactivity of neuroblastoma-binding antibodies.
\end{abstract}

\section{Key words:}

Neuroblastoma, phage display, antibody, hybridoma, Bone marrow 


\section{Introduction:}

Neuroblastoma is the most common extracranial solid tumor in children, accounting for $8-10 \%$ of all childhood cancers. Most patients with neuroblastoma are young (median age at diagnosis is 18 months) and commonly present with metastatic disease. More than $60 \%$ of patients have high-risk tumors with a poor prognosis $^{1}$. Anti-GD2 antibody therapy in patients that have minimal residual disease following stem cell transplantation has led to improvement of survival ${ }^{2}{ }^{3}$. Recently, dinutuximab (a GD-2-binding monoclonal antibody, in combination with granulocyte-macrophage colony-stimulating factor (GM-CSF), interleukin-1 (IL-2), and 13-cis-tetinoic acid (RA) was approved by FDA for pediatric patients with high-risk neuroblastoma.. Anti-GD2 antibody therapy can be associated with considerable pain and its efficacy has only been observed to date in patients with minimal residual disease ${ }^{5}$. Antibody therapy is clearly biologically active but it is important to develop new and better tolerated antibodies to improve the outcomes of patients with neuroblastoma. A multiplicity of antibodies may need to be developed to effectively treat neuroblastoma. At the time of initiating treatment, variable target antigen density on neuroblastoma cells indicates that sensitivity to a single antibody would not be equal for all tumor cells. In addition, regrowth following an initial response may lead to tumor cells that no longer express sufficient target antigen to be inhibited. Therefore, additional treatments with the initial antibody would be ineffective for this new population of tumor cells. Antigen density can be readily assessed with immunohistochemistry and could guide selection of suitable antibodies to treat neuroblastoma if a multiplicity of antibodies were available. Our goal is to use a patient's own set of antibodies that have been generated in response to their tumor to identify new therapeutic antibodies. Therapeutic antibodies generated from a patient to their own tumor may also have applicability to subsequent patients.

Phage display is a powerful method of generating and screening large numbers of antibodies for binding to targets. Using patient-derived B cells to generate phage antibody libraries has the potential advantage of including antibodies that have been made by the patient in response to their own cancer. Phage antibody libraries have been generated from patients with breast cancer, colorectal cancer and multiple tumor types 6789 10111213 including neuroblastoma ${ }^{14}$. Application of these libraries has been primarily to screen for ligands to cancer cell lines or heterologous cancer tissue. Very few reports have described screening an antibody library

against autologous tissue ${ }^{15}$. Using high-resolution liquid chromatography/tandem MS proteomic analyses of serum antibodies coupled with next-generation sequencing of the $\mathrm{V}$ gene repertoire in peripheral B cells, recent analysis of the serum antibody repertoire generated in response to a tetanus toxoid vaccine indicates very little overlap of antibody sequences between individuals ${ }^{16}$. This indicates that the range of antibodies between 
patients will be highly variable even to a common antigenic target. Given the complexity of an endogenous tumor and the molecular variation of tumors between patients, even greater variability of antibodies can be expected. Indeed, between patients there may be little to no overlap of antibodies targeting heterologous tumors. Seeking antibodies that target autologous tumor may be the most productive way to identify therapeutic antibodies.

In this study we took advantage of the availability of residual bone marrow aspirates that were obtained for clinical purpose in pediatric patients with neuroblastoma. We used bone marrow B cells as a source for generating antibodies, using phage display and hybridoma technologies. We also recovered neuroblastoma cells from the same marrow aspirates that allowed screening for the antibodies to autologous cancer cells.

\section{Material and Methods:}

2.1 Patients and tissue collection: Tissue from pediatric patients with neuroblastoma were obtained after an IRB approved consent obtained from each subject or subject guardian. Marrow samples were obtained as part of regular clinical evaluation and residual marrow used for this study.

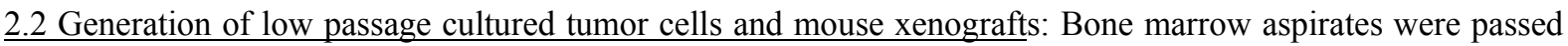
through a $40 \mu \mathrm{m}$ filter to collect neuroblastoma cells as clusters called neurospheres (Fig1, step 1). Retained neuroblastoma cells were cultured with media optimized for the recovery of neuroblastoma cells (Fig1, step 3) ${ }^{17}$. After neuroblastoma cells attained logarithmic growth in tissue culture, cells were expanded. At low passage number, aliquots were cryopreserved. For each sample, the neuroblastoma cell type was confirmed by immunofluorescence using the neuronal markers nestin, synaptophysin, GD2, p75, tyrosine hydroxylase (TH), and NB84. Cultured neuroblastoma cells were implanted subcutaneously in the flanks of NOD-scid IL2Rnull mice (Fig1, step 3). Tumors that grew successfully were engrafted serially in additional mice and also plated for post-xenograft in vitro culture.

2.3 Isolation of marrow mononuclear cells (MNCs): The marrow mononuclear cells were separated from the marrow filtrates using Ficoll density gradient centrifugation. The cells were cryopreserved for the generation of antibodies using phage display and hybrodoma techniques. 
2.4 Generation of phage display scFv library: To conserve limited material, phage libraries were constructed from pooled patient MNCs. Library NB2 was constructed from MNCs pooled from four patients and NB3 library was constructed from MNCs pooled from two patients. Total RNA was extracted from cryoperserved marrow MNCs and pooled from multiple patients for the construction of phage display libraries (Fig1, step 2). cDNA was synthesized from $20 \mu \mathrm{g}$ total RNA using the Superscript III first-strand synthesis system (Invitrogen. Grand Island, NY) with a combination of random hexamers and oligo dT primers to ensure broad representation of antibodies. Each V family of variable regions (VH or VL) was amplified by independent PCR, with a total of 45 different reactions according to previously published methods ${ }^{18}$. A PCR-overlapping process was performed to join both V domains through a linker. The DNA segments encoding the assembled products were then fused to the pIII gene of the pComb3XSS phagemid vector ${ }^{19}$. Following the library construction, 48 to 96 clones were sequenced and analyzed for diversity of $\mathrm{V}$ gene families and variable region amino acid diversity using V-base DNA Plot software.

2.5 Selection of anti-neuroblastoma phage-antibodies: Phage scFv library NB2 was used to select phage antibodies to 0.5 to $2.0 \times 10^{6}$ primary cultured neuroblastoma cells derived from patient MGT-003. Bone marrow MNCs from Patient MGT-003 were included in the set of MNCs used to construct the NB2 library. Phage scFv library NB3 was used to select phage antibodies to neuroblastoma xenograft tissue derived from patient MGT-011. Bone marrow MNCs from patient MGT-011 were included in the set of MNCs used to construct the NB3 library. Thus, phage clones were present in the library used to pan against cell material from the same patient. There was insufficient clinical material to pan against neuroblastoma cells from all of the patients whose MNCs contributed to library construction.

After each round of panning, unbound phages were rinsed off and the cell-bound phages recovered by TG1 bacterial infection. The panning output was amplified and used for the next round of panning. Two to three rounds of panning were performed and individual clones were amplified, normalized by chemititration ${ }^{20}$ and assessed for binding using immunofluorescence (IF) microscopy.

\subsection{Binding analysis of phage clones by immunofluorescence microscopy}

Low passage neuroblastoma cells grown in 96 wells or neuroblastoma xenograft tissue sections were fixed with 3\% paraformaldehyde (PFA). After blocking with imaging-iT FX signal enhancer (Invitrogen, Grand Island, 
$\mathrm{NY}$ ), the cells and sections were incubated with $1 \times 10^{\wedge} 7$ phage particles $/ \mathrm{ml}$. Bound clones were visualized using mouse anti-M13 antibody (GE Health, Pittsburgh, PA) and goat anti-mouse IgG secondary antibody conjugated with Alexa Fluor 568 or Alexa Fluor 488 (Invitrogen, Grand Island, NY). Nuclei were counterstained with 1:1000 diluted DAPI (Invitrogen, Grand Island, NY). The stained cells/tissues were evaluated on Nikon eclipse Ti fluorescent microscope (AVON, MA). The unselected phage libraries and wild type phage (KM13 helper phage) were used as negative controls. Clones that demonstrated binding to neuroblastoma samples were further evaluated for binding to a panel of normal human tissues using the same staining methods described above.

\subsection{Binding analysis of scFv antibodies by Immunofluorecence microscopy:}

The production and purification of soluble scFv antibody of phage clones that showed positive binding on neuroblastoma were done by the method described previously ${ }^{21}{ }_{-}$To evaluate the specific binding of purified $\mathrm{scFv}$ to NB tumor xenograft tissue, scFvs were incubated with tissue sections for 1 hour at room temperature. Slides were rinsed 3 times in PBS and incubated with Alexa Fluor 488 conjugated anti-His tag antibody (Upstate Temecula, CA). The slides were analyzed under fluorescence microscope.

2.8 Hybridoma generation and evaluation of antibodies: MNCs from the marrow sample of patient MGT-003 were used to generate hybridomas (Fig1, step 4). MNCs were stimulated for 3 to 5 days with phytohemagglutinin-L (PHA-L) (Roche, Indianapolis, IN), $2.5 \mu \mathrm{g} / \mathrm{ml}$ ) at $37^{\circ} \mathrm{C} / 5 \% \mathrm{CO}_{2}$ before fusion ${ }^{22}$. $\mathrm{MNCs}$ were fused with murine plasmacytoma P3X63.Ag8.653 (ATCC, Manassas, VA) ${ }^{23}$ under hypo-osmolar condition $^{24}$ using a Multiporator/Helix chamber (Eppendorf, Westbury, NY). The fused cells were distributed in 96-well plates (Corning, Tewksbury, MA) at a density that would yield approximately 0-1 growing hybridomas per well. A total of 360 wells were plated. Following hypoxanthine-aminopterin-thymidine (HAT) (ATCC, Manassas, VA) selection, hybridomas were shifted to 1x hypoxanthine (HT) medium (ATCC, Manassas, VA), and eventually to complete growth medium (RPMI 1640 with 10\% FBS, non-essential amino acids 100x, streptomycin/penicillin 100x, 2-mercaptoethanol 1000x). Antibody production from wells with proliferating cells was determined using ELISA method as follows. Antibody in supernatants were captured with goat anti-human Ig antibody and their levels were determined with anti-Human $\operatorname{IgA} / / \operatorname{IgG} / \operatorname{IgM}(\mathrm{H}+\mathrm{L})-\mathrm{HRP}$ conjugated antibody (Southern Biotech, Birmingham, Alabama). Antibodies were individually assessed for binding to adhered neuroblastoma cells or neuroblastoma xenograft tissue sections derived from the same patient from which the hybridomas were generated. The cells or tissue samples were fixed with $3 \%$ PFA and 
blocked with imaging-iT FX signal enhancer blocker and incubated with the supernatants from individual hybridoma clones. Positive binding was visualized with donkey anti-human Ig-Alexa 488 conjugated antibody (Jackson Immunoreseach, West Grove, PA). Nuclei were stained with DAPI. For staining of free (non-adhered) NB cells, we placed fixed cells into polycarbonate membrane transwell inserts with $5 \mu \mathrm{m}$ membrane diameter in 24 well plate (Corning, Tewksbury, MA). The removal of incubating reagents and rinsing were performed by spinning the inserts in 24 wells at $2000 \mathrm{rpm}$ for 1 minute. $250 \mu 1$ hybridoma supernatant were incubated with cells in inserts at $4{ }^{\circ} \mathrm{C}$ overnight. The positive binding of human antibody to NB cells were visualized by Alexa Fluor 488-conjugated donkey anti-human Ig (Jackson Immunoreseach, West Grove, PA). DAPI was used for nucleus counterstaining. The cells were analyzed under fluorescence microscope.

All the clones with positive cell-binding were further evaluated for Ig subtype and for binding to a panel of normal human tissues.

\section{Results:}

$\underline{3.1 \text { Establishment of primary neuroblastoma cell culture and mouse xenograft model: Four primary cultured }}$ neuroblastoma cell lines derived from the study patients grew sufficiently well to provide adequate samples for our experiments. Also, these four cultured neuroblastoma cell lines achieved serial passage in mice. The tumors in mice provided ample xenograft tissue for the screening.

3.2 Generation of phage scFv library: Two phage display libraries (NB2, and NB3) were generated from patients' bone marrow MNCs. NB2 library was constructed from pooled MNCs from four patients. The number of transducing units (TU) obtained after initial transduction was $2.83 \times 10^{8}$. NB3 library was constructed from pooled MNCs from two patients and the number of TUs obtained after initial transduction was $3.19 \times 10^{8}$. Randomly selected individual clones (48 to 96) from each library were sequenced. Sequence results showed that $78 \%$ of clones had full inserts of heavy and light chain genes. All of the individual clones had unique sequences. These results indicate that the quality of the library met standard criteria of size and diversity for phage library construction ${ }^{18}$.

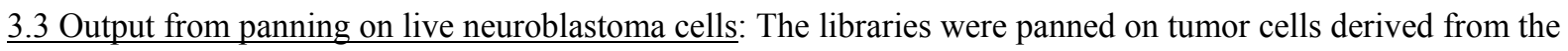
same patients that the libraries were derived. NB2 phage display library was serially panned on living neuroblastoma cells derived from study case MGT-003. From the $3^{\text {rd }}$ panning output, 300 individual clones were 
picked, amplified and binding to MGT-003 cells determined. Six unique clones were identified that bound to MGT-003 cells. Figure 2 showed representative images of phage clones binding to MGT-003 cells. The six clones showed intense binding to some cells, but overall the binding was observed with a minority of the neuroblastoma cell population. This observation was consistent with the heterogeneity of neuroblastoma cells. Five of the 6 clones appeared to bind the cell membrane. The clone that bound to cytoplasmic targets was shown in Fig 2 (clone 2870). This clone appeared to bind a cytoplasmic structure near the nucleus. Of the five clones that appeared to have membrane binding, one representative clone was shown in Figure 3 with a series of confocal images. These six clones were evaluated for binding to a panel of normal human tissues. As shown in table 1, variable binding to normal tissues was observed with these clones.

3.4 Output from panning on neuroblastoma xenograft tissue: The NB3 phage display library was also panned on frozen neuroblastoma xenograft tissue derived from study patient MGT-011. Two hundred clones were randomly selected from $2^{\text {nd }}$ panning output and individual clones assessed for binding to MGT-011 tumor xenograft tissue sections. Six clones bound tumor xenograft tissue as shown in Figure 4. DNA sequence analysis determined that the 6 binding clones were unique. These six clones were evaluated for binding to a panel of normal human tissues including skin, kidney and spleen and normal mouse tissues including kidney and heart. One of the 6 clones bound to all of the normal human and mouse tissues. The other five clones showed no binding above control to any of the normal human or mouse tissues.

Single chain antibodies were prepared from the 6 tumor-binding clones derived from xenograft panning. Four of these 6 clones produced sufficient levels of scFv antibody for binding analyses. After scFv purification, binding of these scFv antibodies were evaluated on patient derived xenograft tumor tissue. All four clones bound to the neuroblastoma xenograft.

3.5 Binding of hybridoma-derived monoclonal antibodies to autologous neuroblastoma tumor cells: A total of 26 wells out of the 360 wells plated produced sufficient antibody for binding analyses. Supernatants of these 26 hybridoma clones were evaluated for binding to the patient-derived mouse xenograft tissues. Four of these 26 antibodies bound to neuroblastoma xenograft tissue (representative images in Fig 5, part A). Sufficient antibody was available to further evaluate two of these 4 xenograft-positive antibodies for binding to low passage neuroblastoma cells from the same patient in a polycarbonate membrane transwell inserts. Both of the 
monoclonal antibodies showed binding to the autologous neuroblastoma cells (Fig 5, part B). ELISA analysis showed these two clones (83G9 and 84D5) were human IgM. Further evaluation of these two clones on a panel of human normal tissues showed one of them have no binding to the tissues tested, while another one showed binding to several normal human tissues.

\section{Discussion:}

Patients with relapsed neuroblastoma will have tumor spread to the bone marrow in a majority of the cases. Given that patient evaluations include bone marrow aspirate to evaluate disease, we have confirmed this to be an adequate source for isolation of neuroblastoma cells, as well as mononuclear cells, without requiring additional biopsies $^{17}$. The cells characterized in culture show differences in antigen expression on the cell surface supporting the need for individualization of antibody targeting.

Phage displayed antibody libraries have been generated from a wide variety of sources. Of particular interest are libraries that have been generated from patients with cancer as the source material. Multiple libraries have been evaluated that were derived from patients with neuroblastoma ${ }^{14}$, breast cancer ${ }^{6}{ }^{7}$, lung cancer $^{8}$, ovarian $_{\text {cancer }}^{9}$ and multiple other tumor types ${ }^{10} 11 \quad 12$ 13. The source material from these patients included peripheral blood and tumor-draining lymph nodes. Since plasma cells reside in the bone marrow this is a potentially attractive source for generating patient-derived antibody libraries. Phage displayed antibody libraries have been generated from the marrow of patients with diseases other than cancer. For example, multiple reports describe application of phage display antibody libraries derived from bone marrow of patients with a variety of autoimmune diseases ${ }^{25} 26$ and infectious diseases such as $\mathrm{HIV}^{28}{ }^{29}$. These marrow-derived libraries have been beneficial to identify clinically relevant antibodies and the cognate antigens. It is anticipated that marrow-derived libraries from patient with cancer will also yield clinically relevant results.

We selected phage-displayed antibodies by panning against low passage autologous tumor cells. Selection of phage displayed antibodies by incubation with whole cells has been extensively reported. This includes cultured cells and freshly harvested cells. This approach has yielded antibodies that selectively bind tumor cells and is a valuable approach for antigen discovery ${ }^{11}{ }^{30}$. However, panning with a phage-displayed antibody library derived from a patient against their own tumor cells has been technically challenging and there are very limited reports describing this approach. Using antibody libraries derived tumor-draining lymph nodes and from tumor 
infiltrating B cells of a patient with breast cancer, we previously reported the identification of multiple antibody clones that bound autologous tumor cells. Interestingly we observed that of the autologous tumor-binding clones, there were multiple instances of shared clonality between antibodies derived from tumor infiltrating B cells and from the lymph node ${ }^{15}$. This current report on neuroblastoma appears to be the first report of identifying phage-displayed antibodies derived from the marrow of patients that bound autologous neuroblastoma cells. Bone marrow biopsies are commonly performed in patients with advanced or recurrent neuroblastoma and may provide a rich resource of autologous antibodies without additional tissue collection.

One of the important challenges to panning on autologous tumor cells is that freshly harvested tumor cells do not grow reliably in tissue culture. One option to establish prolonged growth of freshly harvested tumor cells is to implant the harvested cells in immunodeficient mice. We observed that once a neuroblastoma xenograft was established, robust tumor growth was maintained by serial transplantation or by placement in tissue culture. We used this strategy to obtain autologous tissue for panning. Multiple methods have been reported for panning on tissues which includes panning directly on cut tissue sections ${ }^{31}$. We have previously used this approach for panning on human tumors and also used laser capture to select only the tumor cells from the tissue section ${ }^{21}$. The neuroblastoma xenografts used here were composed of densely concentrated neuroblastoma cells providing a good target for antibody selection. In addition, there were no human background cells that could increase undesirable selection of phage antibodies that bound normal tissue. This report is the first to describe panning on autologous neuroblastoma cells in the xenograft format.

Bone marrow of cancer patients is an uncommon source of B cells for generating human hybridomas. Recent reports describe comparing phage displayed antibodies with hybridoma derived antibodies. These reports observed different binding epitopes as well as different binding affinities which for some targets resulted in phage antibody clones having a lower binding affinity than hybridomas ${ }^{32,33}$. The variable results obtained between these two methods support our attempt to use both phage display and hybridoma technology with these neuroblastoma patients. The rationale against using bone marrow MNCs is that plasma cells, although possessing the desirable property of being affinity matured, are terminally differentiated and therefore will not be an ideal fusion partner using conventional hybridoma methods. We chose to pursue making hybridomas because of the availability of bone marrow MNCs and the unique opportunity to screen monoclones against autologous neuroblastoma tumor cells. The results demonstrated that multiple hybridomas were generated that 
secreted monoclonal antibodies that bound autologous neuroblastoma cells. Two of the antibodies were evaluated for cross binding to normal tissue and did not bind to most of the normal tissue panel. This appears to be the first report to use bone marrow MNCs from neuroblastoma patients to generate antibodies that bind their own tumor. The targets of these antibodies are currently unknown. Preliminarily, hybridoma clones 83G9 and 84D5 and phage clones that bound neuroblastoma xenograft tissue did not have the same binding profile as a commercial anti-GD2 antibody on GD2 positive cells indicating that these hybridoma and phage clones bind targets other than GD2 (data not shown). It also remains to be determined whether these antibodies are bioactive and whether this strategy will be generally applicable in more patients with neuroblastoma.

\section{Conclusion:}

This work evaluated the feasibility of using residual clinical material from pediatric neuroblastoma patients to generate antibodies to autologous tumor. Both the MNCs and the tumor cells were obtained from bone marrow aspirates. Since marrow aspirates are clinically indicated and commonly performed in this patient population, marrow aspirates are readily available and no additional tissue collection events are required to obtain tumor or MNCs. Phage display antibody libraries and hybridomas were generated from the marrow samples. Each method yielded antibodies that bound autologous tumor with some selectivity. It appears feasible to generate human antibodies from residual marrow specimens that bind autologous neuroblastoma cells. Expansion of this approach is underway to collect more specimens, optimize methods to generate antibodies, and to evaluate the function of neuroblastoma-binding antibodies.

Acknowledgements: This study was supported by the Department of Defense Congressionally Directed Medical Research Program U.S. Army grant W81XWH-12-1-0332, and in part by SD Ireland Professorship of Oncology for Research and Beat NB Cancer Foundation

Conflict of interest: The authors of this paper report no conflict of interest with regard to this paper.

\section{Reference:}

1. Navid F, Armstrong M, Barfield RC. Immune therapies for neuroblastoma. Cancer biology \& therapy 2009;8:874-82.

2. Fish JD, Grupp SA. Stem cell transplantation for neuroblastoma. Bone marrow transplantation 2008;41:159-65.

3. Yu AL, Gilman AL, Ozkaynak MF, et al. Anti-GD2 antibody with GM-CSF, interleukin-2, and isotretinoin for neuroblastoma. The New England journal of medicine 2010;363.

4. Dhillon S. Dinutuximab: first global approval. Drugs 2015;75:923-7.

5. Cheung NK, Dyer MA. Neuroblastoma: developmental biology, cancer genomics and immunotherapy. Nature reviews Cancer 2013;13:397-411. 
6. Rubinstein DB, Stortchevoi A, Boosalis M, Ashfaq R, Guillaume T. Overexpression of DNA-binding protein B gene product in breast cancer as detected by in vitro-generated combinatorial human immunoglobulin libraries. Cancer research 2002;62:4985-91.

7. Belimezi MM, Papanastassiou D, Merkouri E, Baxevanis CN, Mamalaki A. Growth inhibition of breast cancer cell lines overexpressing Her2/neu by a novel internalized fully human Fab antibody fragment. Cancer immunology, immunotherapy : CII 2006;55:1091-9.

8. Graus YF, Verschuuren JJ, Degenhardt A, et al. Selection of recombinant anti-HuD Fab fragments from a phage display antibody library of a lung cancer patient with paraneoplastic encephalomyelitis. Journal of neuroimmunology 1998;82:200-9.

9. Figini M, Martin F, Ferri R, et al. Conversion of murine antibodies to human antibodies and their optimization for ovarian cancer therapy targeted to the folate receptor. Cancer immunology, immunotherapy : CII 2009;58:531-46.

10. Roovers RC, Van Der Linden E, Zijlema H, de Bruïne A, Arends JW, Hoogenboom HR. Evidence for a bias toward intracellular antigens in the local humoral anti-tumor immune response of a colorectal cancer patient revealed by phage display. International journal of cancer Journal international du cancer 2001;93.

11. Baskar S, Suschak JM, Samija I, et al. A human monoclonal antibody drug and target discovery platform for B-cell chronic lymphocytic leukemia based on allogeneic hematopoietic stem cell transplantation and phage display. Blood 2009;114:4494-502.

12. Li J, Pereira S, Van Belle P, et al. Isolation of the melanoma-associated antigen p23 using antibody phage display. Journal of immunology (Baltimore, Md : 1950) 2001;166:432-8.

13. Dantas-Barbosa C, Faria FP, Brigido MM, Maranhao AQ. Isolation of osteosarcoma-associated human antibodies from a combinatorial Fab phage display library. Journal of biomedicine \&amp; biotechnology 2009;2009:157531.

14. Uttenreuther-Fischer MM, Kruger JA, Fischer P. Molecular characterization of the anti-idiotypic immune response of a relapse-free neuroblastoma patient following antibody therapy: a possible vaccine against tumors of neuroectodermal origin? Journal of immunology (Baltimore, Md : 1950) 2006;176:7775-86.

15. Novinger LJ, Ashikaga T, Krag DN. Identification of tumor-binding scFv derived from clonally related B cells in tumor and lymph node of a patient with breast cancer. Cancer immunology, immunotherapy : CII 2014.

16. Lavinder JJ, Wine Y, Giesecke C, et al. Identification and characterization of the constituent human serum antibodies elicited by vaccination. Proceedings of the National Academy of Sciences of the United States of 2014;111.

17. Hansford LM, McKee AE, Zhang L, et al. Neuroblastoma cells isolated from bone marrow metastases contain a naturally enriched tumor-initiating cell. Cancer Res 2007;67:11234-43.

18. Andris-Widhope J, et al.,. Generation of Antibody Libraries: PCR Amplification andAssembly of Ligh- and Heavy-chain Coding Sequences,. In: B. Barbas S, Silverman, ed. Phage Display: A LaboratoryManual. Cold Spring Harbor: Cole Spring Harbor Laborary Press; 2001:p. 9.1-9.35.

19. Scott JKaCFB. Phage-display Vectors,. In: C.F. Barbas ea, ed. Phage Display: A LaboratoryManual. New York Cold Spring Harbor Laboratory Press; 2001:p. 2.1-2.19.

20. Shukla GS, Krag DN. Selection of tumor-targeting agents on freshly excised human breast tumors using a phage display library. Oncology reports 2005;13:757-64.

21. Sun Y, Shukla GS, Weaver D, Pero SC, Krag DN. Phage-display selection on tumor histological specimens with laser capture microdissection. Journal of immunological methods 2009;347:46-53.

22. Schmidt E, Leinfelder U, Gessner P, Zillikens D, Brocker EB, Zimmermann U. CD19+ B lymphocytes are the major source of human antibody-secreting hybridomas generated by electrofusion. Journal of immunological 
methods 2001;255:93-102.

23. Kearney JF, Radbruch A, Liesegang B, Rajewsky K. A new mouse myeloma cell line that has lost immunoglobulin expression but permits the construction of antibody-secreting hybrid cell lines. J Immunol 1979;123:1548-50.

24. Zimmermann U, Gessner P, Schnettler R, Perkins S, Foung SK. Efficient hybridization of mouse-human cell lines by means of hypo-osmolar electrofusion. J Immunol Methods 1990;134:43-50.

25. Fischer $\mathrm{P}$, Jendreyko $\mathrm{N}$, Hoffmann $\mathrm{M}$, et al. Platelet-reactive IgG antibodies cloned by phage display and panning with IVIG from three patients with autoimmune thrombocytopenia. British journal of haematology 1999;105:626-40.

26. Maruyama T, Saito I, Hayashi $Y$, et al. Molecular analysis of the human autoantibody response to alpha-fodrin in Sjogren's syndrome reveals novel apoptosis-induced specificity. The American journal of pathology 2004;165:53-61.

27. Farnaes L, Ditzel HJ. Dissecting the cellular functions of annexin XI using recombinant human annexin XI-specific autoantibodies cloned by phage display. The Journal of biological chemistry 2003;278:33120-6.

28. Nelson JD, Kinkead H, Brunel FM, et al. Antibody elicited against the gp41 N-heptad repeat (NHR) coiled-coil can neutralize HIV-1 with modest potency but non-neutralizing antibodies also bind to NHR mimetics. Virology 2008;377:170-83.

29. Bjorling E, von Garrelts E, Morner A, Ehnlund M, Persson MA. Human neutralizing human immunodeficiency virus type 2-specific Fab molecules generated by phage display. The Journal of general virology 1999;80 ( Pt 8):1987-93.

30. Jakobsen CG, Rasmussen N, Laenkholm AV, Ditzel HJ. Phage display derived human monoclonal antibodies isolated by binding to the surface of live primary breast cancer cells recognize GRP78. Cancer Res 2007;67:9507-17.

31. Jarutat T, Nickels C, Frisch C, et al. Selection of vimentin-specific antibodies from the HuCAL phage display library by subtractive panning on formalin-fixed, paraffin-embedded tissue. Biological chemistry 2007;388:651-8.

32. Sorouri M, Fitzsimmons SP, Aydanian AG, Bennett S, Shapiro MA. Diversity of the antibody response to tetanus toxoid: comparison of hybridoma library to phage display library. PloS one 2014;9.

33. Rossant CJ, Carroll D, Huang L, et al. Phage display and hybridoma generation of antibodies to human CXCR2 yields antibodies with distinct mechanisms and epitopes. mAbs 2014;6:1425-38. 


\section{Figure and table legends:}

Fig 1: Schematic presentation of methods used in this project. Neuroblastoma patients' bone marrow samples (step 1) were used for tumor cell isolation and mononuclear cells (MNC) separation. Selected MNC population were used for phage display scFv library construction (step 2) and human hybridoma cells generation (step 4). Isolated tumor cells (step 3) were used for in vitro tissue culture and mouse xenograft modal establishment. The generated phage libraries were used for panning and screening on matching patients' primary cultured tumor cells and/or mouse xenograft tissue sections using immunofluorescence (IF) staining (step 5). Monoclonal human antibodies selected from hybridoma work were tested on matching patients' in vitro cultured tumor cells and/or mouse xenograft tissues (step 6). Tumor specific antibodies were identified in step 7 by screening on normal human tissues by IF stain.

Fig 2: Immunofluorescence (IF) analysis of six phage clones showing positive binding to primary cultured MGT-003 tumor cells. PFA-fixed cultured MGT-003 cells were incubated with $1 \times 10^{12}$ TU phage samples of each clone. Positive bindings were visualized using mouse anti-M13 antibody and goat-anti-mouse IgG -Alex568 secondary antibody (red signal). Nucleus were counterstained with DAPI (blue signal).

Fig 3: Confocal microscopy observation of phage clone 2711 binding to MGT-003 NB patient's primary cultured tumor cells. Cells cultured on $12 \mathrm{~mm}$ poly-lysate slides were fixed in 3\% PFA and stained with phage clones and followed with mouse anti-M13 antibody and goat-anti-mouse IgG -Alex568 secondary antibody (red signal). Nucleus were counterstained with DAPI (blue signal). Z layers images were captured from top to bottom.

Fig 4: Immunofluorescence (IF) analysis of six phage clones that show positive binding to MGT-011 xenograft tissue. Frozen tissue sections fixed with 3\% PFA were stained with phage clones following with mouse anti-M13 antibody and goat-anti-mouse $\operatorname{IgG}-\mathrm{Alex} 568$ secondary antibody (red signal). Nucleus were counterstained with DAPI (blue signal).

Fig 5: Selected clones' hybridoma supernatant IF stain on MGT-003 xenograft tissue (part A) and MGT-003 whole cells in filter cups (part B). Positive signals (green) were visualized by donkey anti-human IgM-Alex 488 conjugated antibody. Top row of images were captured positive signal (green). Bottom row of 
images were stained by DAPI (blue) to show the structure of tissue and cells.

Table 1: Imaging analysis results of selected neuroblastoma cells and xenograft tissue binding phage clones and hybridoma clones on a panel of normal human tissue. "++" means strong binding to tissue. "+" means weak binding to tissue. “-” means negative binding to tissue. 


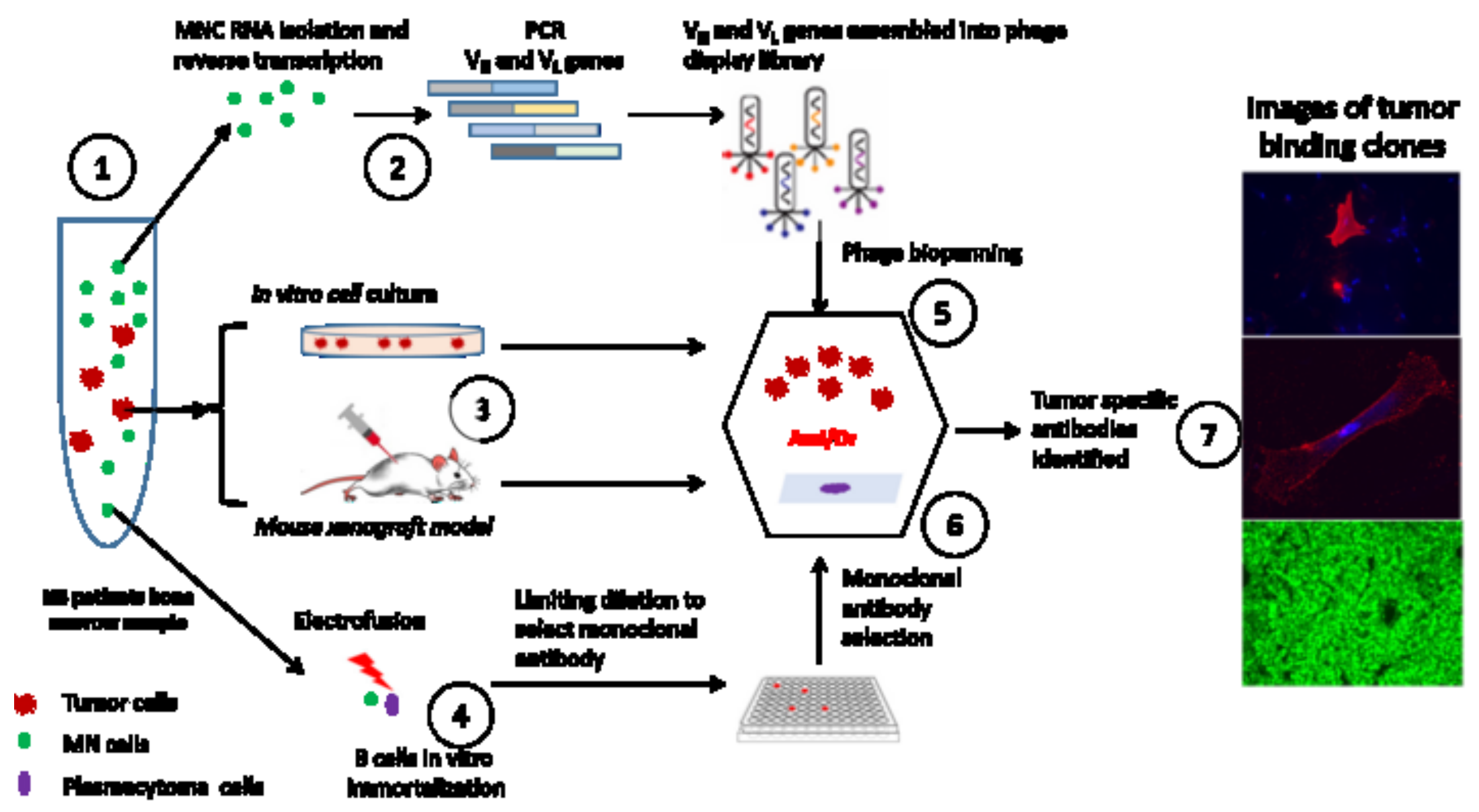




\begin{tabular}{|c|c|c|c|c|c|c|}
\hline Group & Clone No. & Skin & Kidney & Spleen & Liver & Uterine \\
\hline \multirow{6}{*}{$\begin{array}{l}\text { Phage clones } \\
\text { selected on in } \\
\text { vitro primary } \\
\text { cultured NB } \\
\text { cell }\end{array}$} & 2711 & - & - & ++ & + & - \\
\hline & 2718 & - & - & + & + & - \\
\hline & 2844 & - & - & - & + & - \\
\hline & 2870 & - & - & - & - & - \\
\hline & 2929 & - & + & ++ & + & - \\
\hline & 3047 & - & - & - & - & - \\
\hline \multirow{6}{*}{$\begin{array}{c}\text { Phage clones } \\
\text { selected on } \\
\text { mouse } \\
\text { xenograft } \\
\text { tissues }\end{array}$} & 1100-A7 & - & - & - & $\mathrm{N} / \mathrm{A}$ & $\mathrm{N} / \mathrm{A}$ \\
\hline & 1100-B7 & - & - & - & $\mathrm{N} / \mathrm{A}$ & $\mathrm{N} / \mathrm{A}$ \\
\hline & $1100-\mathrm{C3}$ & - & - & - & $\mathrm{N} / \mathrm{A}$ & $\mathrm{N} / \mathrm{A}$ \\
\hline & $1100-C 10$ & - & - & - & $\mathrm{N} / \mathrm{A}$ & $\mathrm{N} / \mathrm{A}$ \\
\hline & 1100-D6 & - & - & - & $\mathrm{N} / \mathrm{A}$ & $\mathrm{N} / \mathrm{A}$ \\
\hline & $1100-\mathrm{H} 7$ & + & + & + & $\mathrm{N} / \mathrm{A}$ & $\mathrm{N} / \mathrm{A}$ \\
\hline \multirow{2}{*}{$\begin{array}{l}\text { Hybridoma } \\
\text { clones }\end{array}$} & $83 G 9$ & - & - & - & - & - \\
\hline & 84D5 & - & + & - & ++ & - \\
\hline
\end{tabular}

Table 1: Imaging analysis results of selected neuroblastoma cells and xenograft tissue binding phage clones and hybridoma clones on a panel of normal human tissue. "++" showed strong binding to tissue. "+" showed weak binding to tissue. "-" showed negative binding to tissue. 


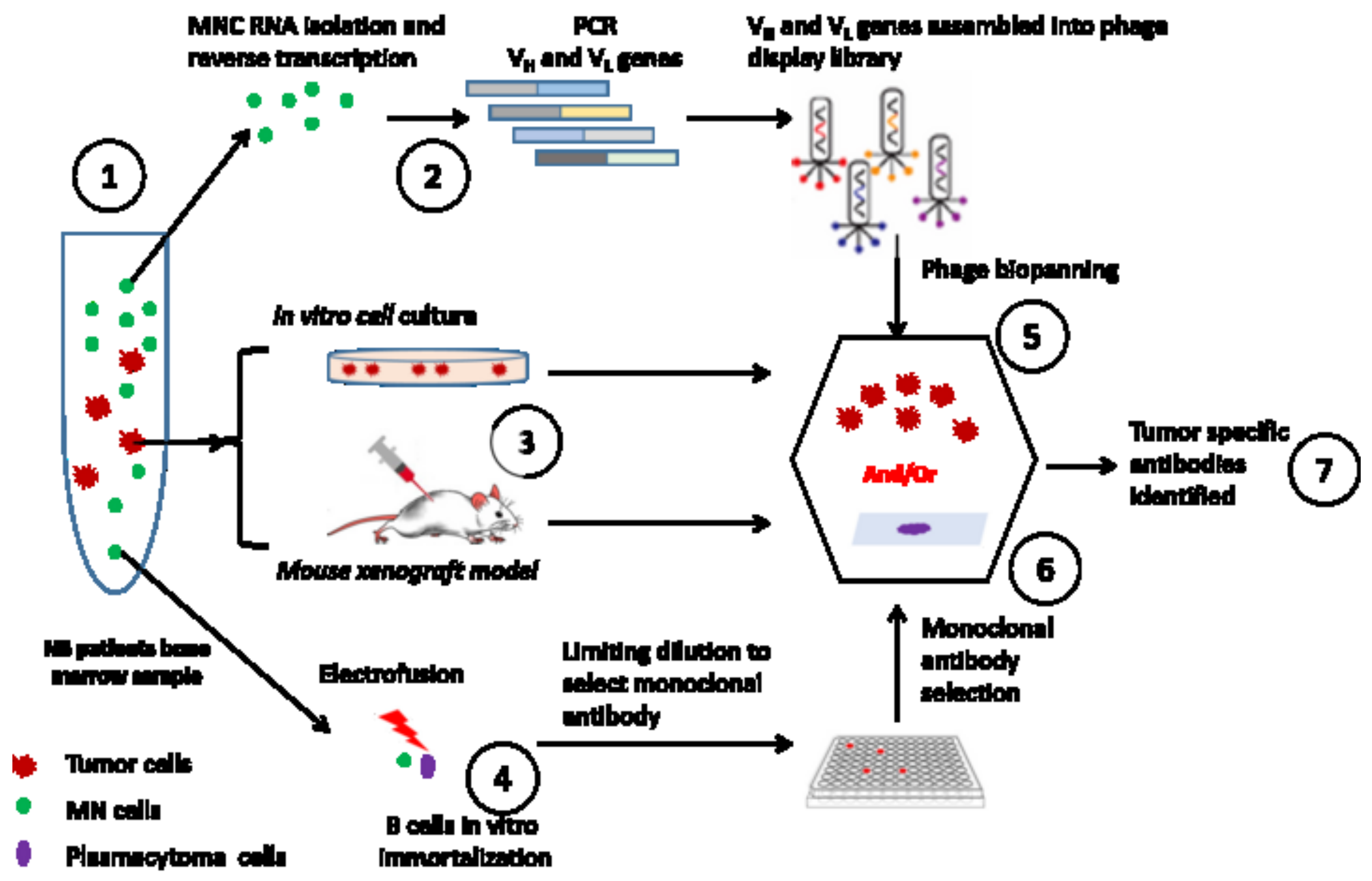




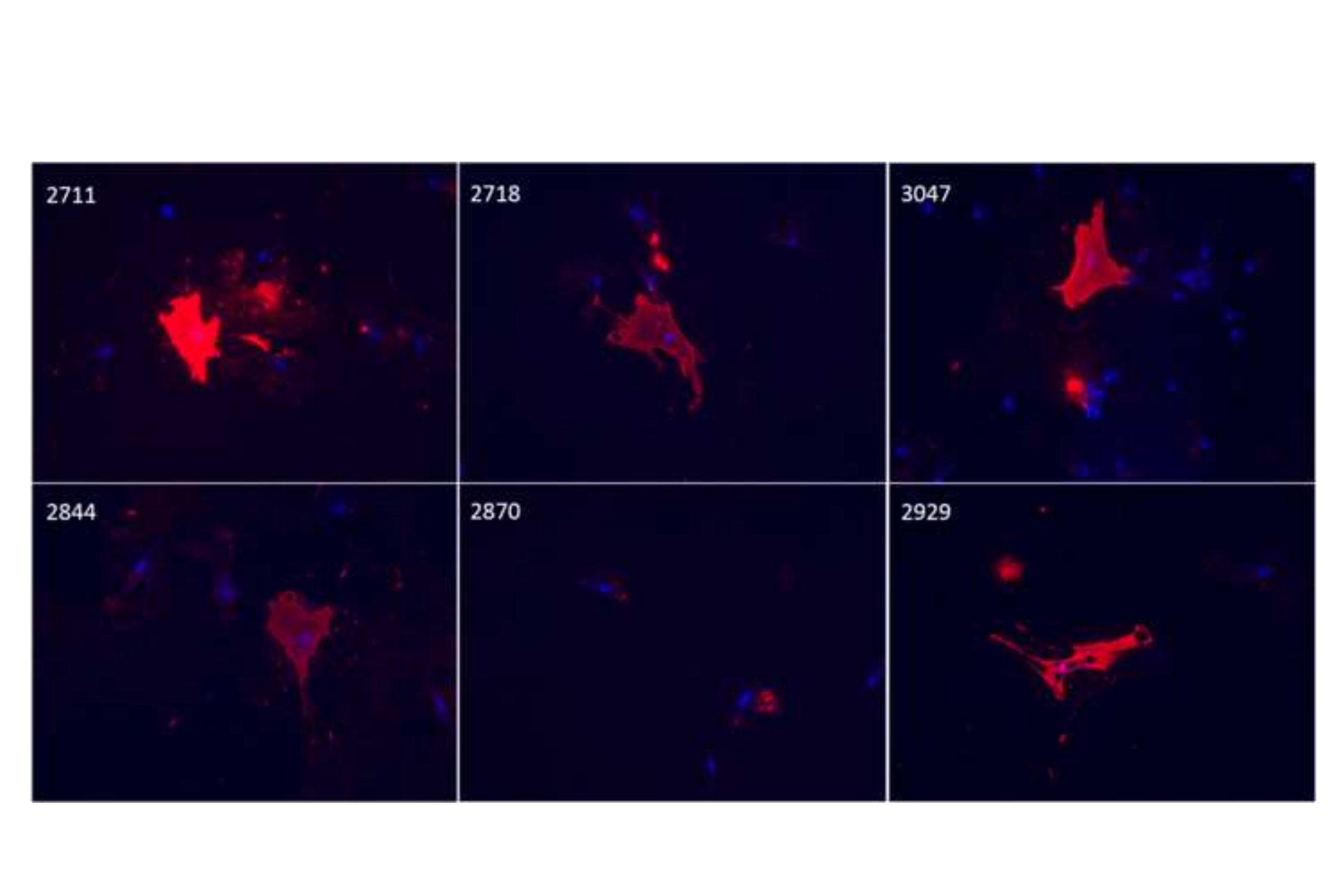

(

(1)

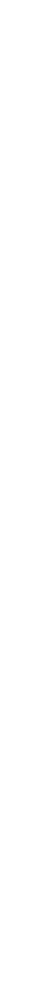

(1)

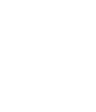

Figure

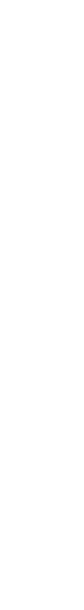

.
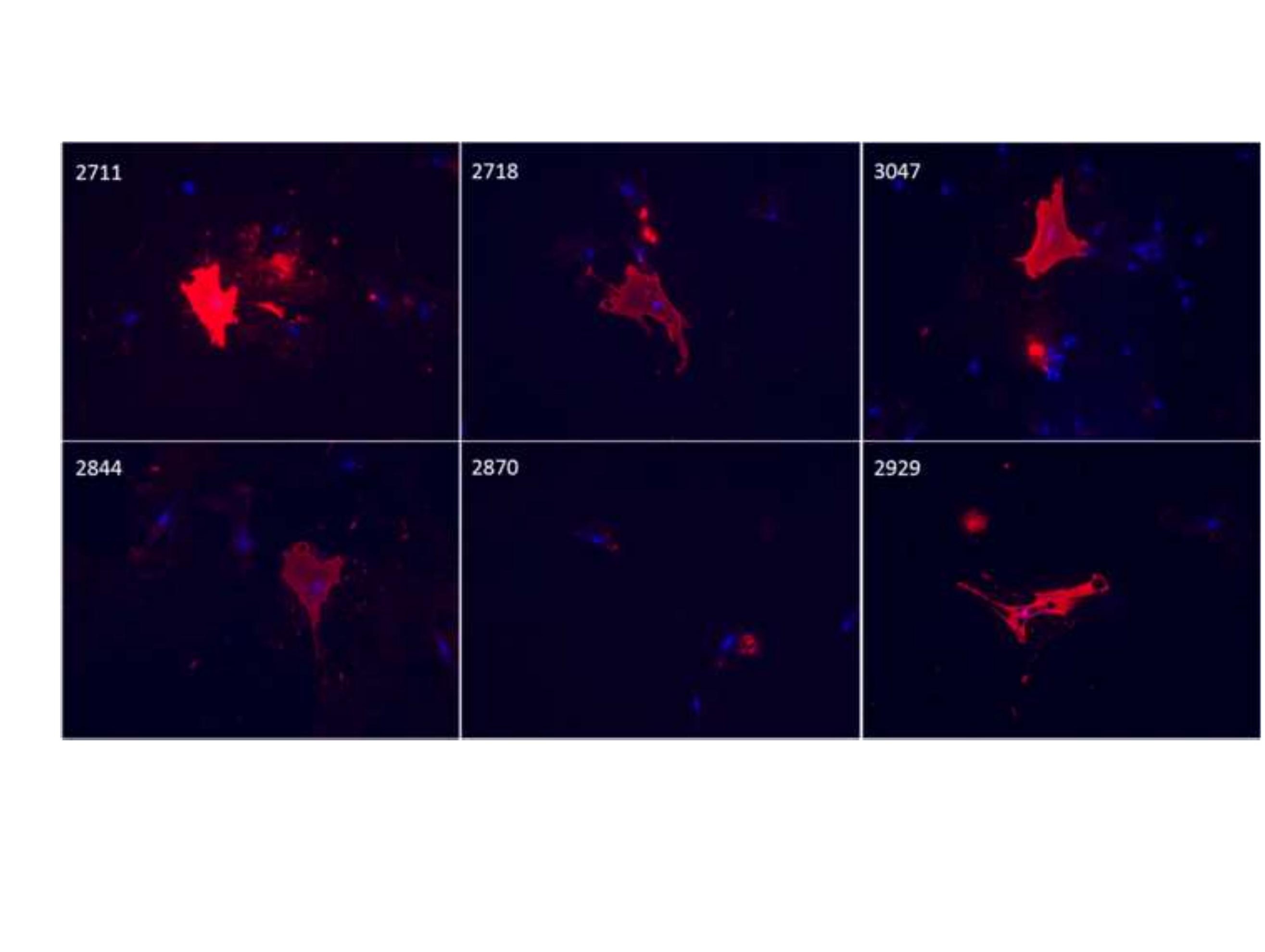
Layer 1

Layer 2

Layer 3

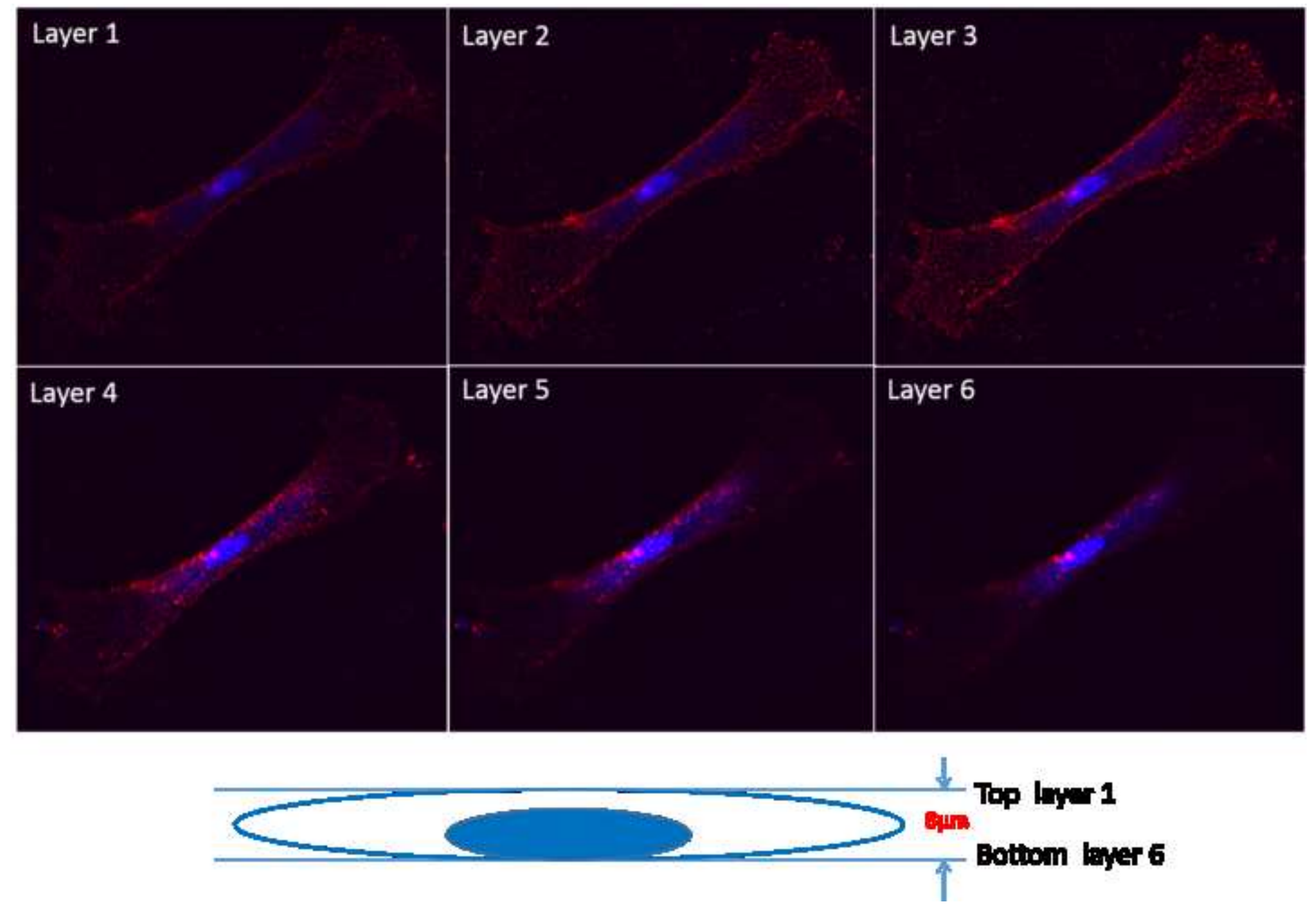



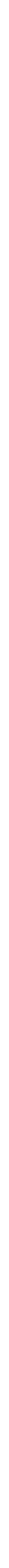